Arq. Bras. Med. Vet. Zootec., v.67, n.1, p.255-264, 2015

\title{
Cultivo do peixe ornamental molinésia (Poecilia sp.) em esgotos domésticos tratados: desempenho zootécnico e avaliação do bem-estar animal
}

\author{
[Cultivation of ornamental molly (Poecilia sp.) fish in treated sewage: zootechnical performance \\ and animal welfare evaluation] \\ E.S. Santos ${ }^{1}$, S. Mota $^{2 *}$, A.B. Santos ${ }^{2}$, M.D. Aquino ${ }^{2}$ \\ ${ }^{1}$ Instituto Federal de Educação - Ciência e Tecnologia do Ceará - IFCE - Fortaleza, CE \\ ${ }^{2}$ Universidade Federal do Ceará - UFC - Fortaleza, CE
}

\begin{abstract}
RESUMO
Neste trabalho estudou-se a utilização de esgotos domésticos tratados na criação do peixe ornamental molinésia (Poecilia sp.), observando o desempenho zootécnico e avaliando-se o bem-estar dos peixes cultivados. Foram testados três tratamentos: ET - abastecido com esgoto tratado em lagoas de estabilização; $\mathrm{ED}$ - abastecido com esgoto tratado e água de poço diluídos em iguais proporções; $\mathrm{AB}$ abastecido com água de poço. Por meio dos resultados dos parâmetros físico-químicos, observou-se que o esgoto doméstico tratado em lagoas de estabilização oferece condição para se realizar o cultivo da espécie, com melhor desempenho quando diluído em água. Em todos os parâmetros de rendimento zootécnicos avaliados, o tratamento que utilizou o esgoto tratado diluído foi o que apresentou os melhores resultados. Dentre os indicadores de bem-estar observados, apenas a avaliação histopatológica foi capaz de fornecer resultado conclusivo, o qual apontou o tratamento que utilizou esgoto tratado diluído como o que proporcionou maior conforto à espécie cultivada. As características ornamentais dos peixes cultivados não sofreram influência do uso do esgoto doméstico tratado, observando-se que os padrões de coloração e formato de nadadeiras não foram alterados.
\end{abstract}

Palavras-chave: aquicultura ornamental, lagoas de estabilização, molinésia (Poecilia sp.), reúso de água

\begin{abstract}
This work evaluated the treated sewage reuse in ornamental molly fish creation, evaluating the zootechnical performance and animal welfare. Three treatments were tested: ET - supplied with treated sewage; ED - supplied with sewage and raw water in the same proportion; AB - supplied with raw water. The physical-chemical results indicated that the treated sewage from stabilization ponds was adequate for the species cultivation only when diluted in raw water. Among the welfare indicators, only histopathological evaluation was able to provide conclusive results, which showed that the treated sewage diluted treatment was the best in terms of comfort for the cultivated species. The ornamental characteristics of farmed fish were not influenced by the use of treated sewage, in which the patterns of color and shape of fins were not changed.
\end{abstract}

Keywords: ornamental aquaculture, stabilization ponds, molly fish (Poecilia sp.), water reuse

\section{INTRODUÇÃO}

Segundo Bunting (2007), há uma clara e urgente necessidade de que sejam desenvolvidos instrumentos que facilitem a análise de

Recebido em 26 de março de 2014

Aceito em 11 de setembro de 2014

*Autor para correspondência (corresponding author)

E-mail: suetonio@ufc.br alternativas de tratamento de esgoto e opções de reúso, incluindo os aspectos econômicos, financeiros, sociais e institucionais.

No cultivo de peixes destinados à alimentação humana, em esgotos tratados, há o risco de contaminação do tecido comestível. No entanto, 
cultivando-se peixes ornamentais, elimina-se a via de contaminação humana por ingestão do pescado infectado, reduzindo-se os riscos dessa atividade.

É grande o potencial para o desenvolvimento do cultivo de peixes ornamentais. No entanto, ainda são poucos os estudos sobre o uso de esgotos tratados na sua produção.

Com milhares de espécies diferentes de peixes ornamentais, de todas as formas e tamanhos, sendo utilizadas em aquários domésticos, surpreende como poucas pesquisas têm considerado o aspecto de bem-estar dos peixes (Saxby et al., 2010).

Os peixes da espécie Poecilia sp. habitam águas doces e se estabelecem com sucesso em uma variedade de condições ambientais (Sudha, 2012). Existe hoje no mercado grande diversidade de cores e formas de peixes molinésia, razão pela qual têm sido bastante utilizados em aquários residenciais.
Este trabalho teve por objetivo avaliar o desempenho zootécnico e o bem-estar de peixes Poecilia sp cultivados em tanques com esgotos domésticos tratados em lagoas de estabilização.

\section{MATERIAL E MÉTODOS}

Foram utilizados três tanques com $40,00 \mathrm{~m}^{3}$ de volume útil cada, preenchidos com esgoto doméstico, efluente da última lagoa de estabilização de um sistema composto por quatro lagoas em série, sendo uma anaeróbia, uma facultativa e duas de maturação. Em cada tanque foram colocados três tanques-rede (TR) com volume útil de $3,0 \mathrm{~m}^{3}$, confeccionados com tela industrial com abertura de malha de $0,5 \mathrm{~mm}$ e cobertos com tela tipo sombrite $50 \%$.

$\mathrm{Na}$ Figura 1, mostra-se o lay-out da área experimental, indicando-se a disposição dos tanques-rede nos tanques.

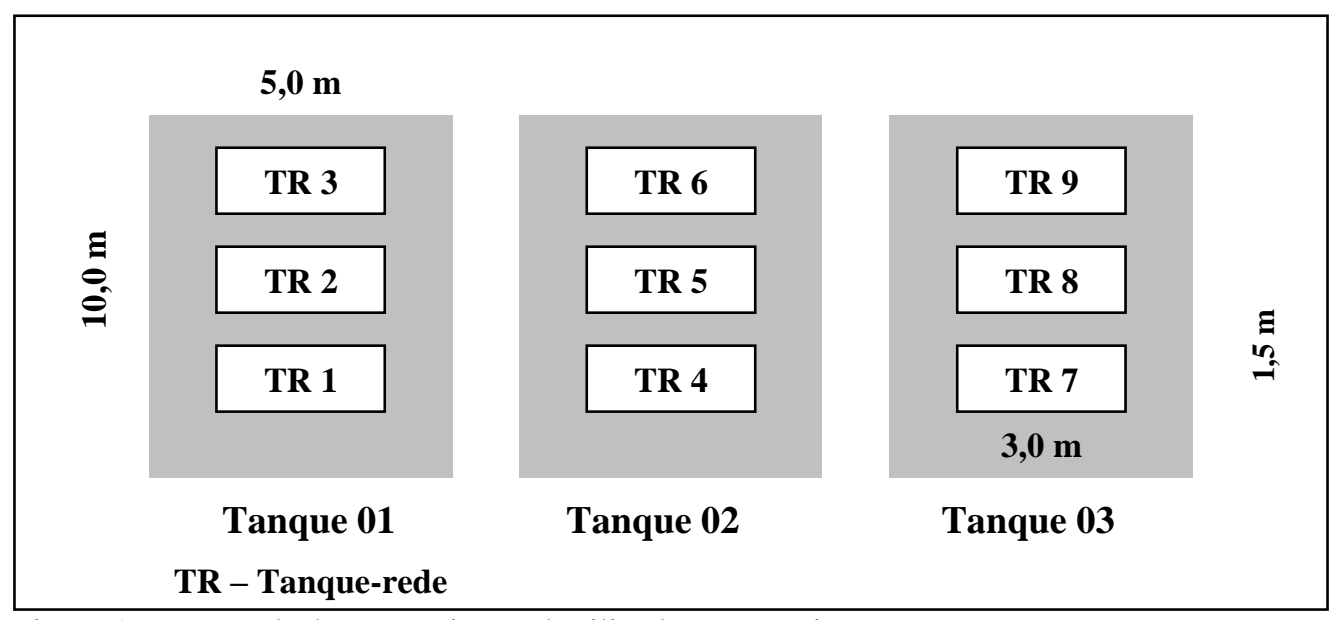

Figura 1. Lay-out da área experimental utilizada na pesquisa.

Foram testados três tratamentos, com três repetições cada, conforme descritos a seguir:

- Tratamento ET $\rightarrow$ Tanque 01 - Tanquesrede 1 (TR-1), 2 (TR-2) e 3 (TR-3): tanque abastecido com esgoto doméstico tratado no sistema de lagoas de estabilização (efluente final);

- $\quad$ Tratamento ED $\rightarrow$ Tanque 02 - Tanquesrede 4 (TR-4), 5 (TR-5) e 6 (TR-6): tanque abastecido com esgoto diluído, sendo a metade do volume constituída de esgoto doméstico tratado e a outra metade, de água de poço;

- Tratamento AB $\rightarrow$ Tanque 03 - Tanquesrede 7 (TR-7), 8 (TR-8) e 9 (TR-9): o tanque foi abastecido com água bruta proveniente de fonte subterrânea; foi fornecida alimentação artificial (ração) neste tanque.

Nos tanques-rede foram estocados alevinos de molinésia Poecilia sp. na densidade experimental de 200 peixes $/ \mathrm{m}^{3}$ (600 peixes/tanque-rede), igualmente nos três tratamentos testados. 
Realizou-se biometria da população inicial piscícola estocada nos tanques experimentais, como indicado na Tabela 1.

Na presente pesquisa foram utilizados peixes da Família Poeciliidae, mais precisamente do subgênero Mollienesia, popularmente conhecidos como molinésias, cultivados em esgoto doméstico tratado em um sistema de lagoas de estabilização.

Tabela 1. Comprimento total médio e peso médio dos peixes no início da pesquisa

\begin{tabular}{lccc}
\hline \multicolumn{1}{c}{ Parâmetro Zootécnico } & \multicolumn{3}{c}{ Tratamentos Experimentais } \\
& ET & ED & AB \\
\hline Comprimento total inicial $(\mathrm{mm})$ & $12,6 \pm 2,50 \mathrm{a}$ & $12,5 \pm 1,81 \mathrm{a}$ & $12,0 \pm 1,79 \mathrm{a}$ \\
Peso médio inicial $(\mathrm{g})$ & $0,032 \pm 0,006 \mathrm{a}$ & $0,033 \pm 0,007 \mathrm{a}$ & $0,032 \pm 0,004 \mathrm{a}$ \\
\hline
\end{tabular}

ET - esgoto tratado; $\mathrm{ED}$ - esgoto diluído; $\mathrm{AB}$ - água bruta.

O experimento teve duração de 56 dias, tendo sido realizadas quatro biometrias. $\mathrm{Na}$ pesagem dos indivíduos, usou-se uma balança digital do tipo Filizola, modelo MF-I, com precisão de $0,5 \mathrm{~g}$. Para as medições de comprimento total dos peixes, utilizou-se um paquímetro digital Western PRO com precisão de $0,1 \mathrm{~mm}$. As análises de temperatura (7 análises) e oxigênio dissolvido (OD - 10 análises) foram monitoradas in loco utilizando-se uma sonda multiparamétrica modelo YSI-55. Para a realização das demais análises, foram coletadas amostras nos três tanques, as quais foram analisadas em laboratório, observando a metodologia constante do Standard Methods (APHA, 2005): pH (7 análises por tanque), condutividade elétrica (5 análises por tanque), alcalinidade total (5 análises por tanque), dureza total (5 análises por tanque), nitrogênio amoniacal total (6 análises por tanque), nitrito (7 análises por tanque), nitrato ( 5 análises por tanque), cloreto (5 análises por tanque) e Demanda Química de Oxigênio (DQO -6 análises por tanque).

Os valores de amônia não ionizada (mg de N$\mathrm{NH}_{3} \mathrm{~L}^{-1}$ ) foram calculados a partir da aplicação dos resultados obtidos de amônia total, $\mathrm{pH}$ e temperatura, usando as Equações 01 e 02, propostas por Albert (1973) e Emerson et al. (1975), respectivamente.

$$
\begin{aligned}
& \mathrm{NH}_{3}=\left[\mathrm{NH}_{3}+\mathrm{NH}_{4}^{+}\right] /\left[1+10^{(\mathrm{pKa}-\mathrm{pH})}\right] \\
& \mathrm{pKa}=0,0918+2729,92 /\left[\mathrm{T}\left({ }^{\circ} \mathrm{C}\right)+273\right]
\end{aligned}
$$

Os parâmetros utilizados para a avaliação do rendimento zootécnico dos tratamentos testados foram: ganho de peso; ganho de peso diário; taxa de crescimento específico diário, crescimento, em comprimento; crescimento diário; taxa de sobrevivência.

Durante as biometrias realizadas (em número de quatro) no decorrer do cultivo, os peixes eram pesados e medidos, sendo possível estimar a relação peso:comprimento e, então, aplicar os fatores de condição alométrico e de Fulton. A relação peso:comprimento foi obtida plotando-se em gráfico o peso médio $(\mathrm{g})$ dos peixes no eixo " $x$ " e o comprimento total $(\mathrm{cm})$ no eixo " $y$ ", sendo gerada a curva que representa a dispersão dos pontos de intersecção entre os dois eixos, representada pela Equação 03 (Santos et al., 2002):

$\mathrm{P}=\quad \mathrm{a} \cdot \mathrm{C}^{\mathrm{b}}$

Sendo: $\mathrm{P}=$ Peso médio $(\mathrm{g})$;

$\mathrm{C}=\quad$ Comprimento total $(\mathrm{cm})$;

$\mathrm{a}=\quad$ Constante de regressão (intercepto);

$\mathrm{b}=$ Coeficiente de regressão (coeficiente angular).

Para estimar o bem-estar dos peixes, foram utilizados os fatores de condição de Fulton (Piedras et al., 2006) e alométrico (Lemos et al., 2006; Almeida e Nuñer, 2009), os quais estão representados nas Equações 04 e 05, respectivamente. Esses fatores devem ser mantidos em valores mais elevados possível e constantes ao longo do ciclo vital (Gomiero e Braga, 2003). 


$$
\begin{aligned}
& \mathrm{K}_{\text {Fulton }}=\left(\mathrm{P} / \mathrm{C}_{\mathrm{t}}^{3}\right) \times 100 \\
& \mathrm{~K}_{\text {alom }}=\left(\mathrm{P} / \mathrm{C}_{\mathrm{t}}^{3}\right) \times 100
\end{aligned}
$$

Sendo:

$\mathrm{K}_{\text {Fulton }}=$ Fator de condição de Fulton;

$3=\quad$ Constante de Fulton, a qual considera crescimento isométrico.

$\mathrm{K}_{\mathrm{alom}}=$ Fator de condição alométrico;

$\mathrm{b}=\quad$ Coeficiente de regressão (coeficiente angular).

O coeficiente de regressão (b) equivale ao coeficiente de alometria $(\theta)$, que, por sua vez, representa a forma de crescimento do indivíduo (Santos et al., 2002). Na Tabela 2, observam-se as possíveis relações entre o coeficiente de alometria $(\theta)$ e as proporções de incremento de comprimento e peso.

Tabela 2. Possíveis relações entre o coeficiente de alometria $(\theta)$ e as proporções de incremento de comprimento e peso

\begin{tabular}{ccc}
\hline Relação & Forma de crescimento & Proporção de incremento \\
\hline$\theta<3$ & Alometria negativa & comprimento $>$ peso \\
$\theta=3$ & Isometria & comprimento $=$ peso \\
$\theta>3$ & Alometria positiva & comprimento $<$ peso \\
\hline
\end{tabular}

Ao final do experimento foram retirados dez peixes, aleatoriamente, de cada tratamento, os quais foram anestesiados por imersão em solução com eugenol (300mg L $\mathrm{m}^{-1}$ ), sacrificados por meio de secção cervical e fixados com solução de Davidson.

A utilização de molinésias, peixes ornamentais de pequeno porte, permitiu que os cortes histológicos abrangessem a total extensão corporal dos espécimes analisados; dessa forma, em cada lâmina foi possível observar todos os órgãos de cada peixe. Para preparação das lâminas, foi utilizado o processamento histológico padrão, conforme descrito em Camargo e Martinez (2007).

Para identificar a influência do meio ambiente no bem-estar dos peixes cultivados, utilizou-se o protocolo proposto por Bernet et al. (1999) para a avaliação dos tecidos amostrados. Essa metodologia avalia os danos ocorridos nos tecidos por meio de índices obtidos a partir de dois atributos numéricos: (1) a importância da alteração patológica definida por intermédio de um fator de importância; (2) a extensão da alteração patológica no tecido quantificada por meio de um escore numérico.
O fator de importância de uma lesão é dado em função do seu efeito sobre a habilidade do peixe em sobreviver (Bernet et al., 1999), enquanto o valor numérico corresponde à extensão da lesão observada em todo o campo visual analisado sob microscopia.

O somatório da multiplicação do fator de importância pelo valor numérico das alterações diagnosticadas em cada um dos órgãos analisados resultou em diferentes valores desse índice para cada um dos órgãos (de dez peixes retirados aleatoriamente, de cada tratamento), o que permitiu as análises estatísticas.

Os dados de crescimento em comprimento, ganho de peso e ganho de peso diário, da taxa de crescimento específico, fatores de condição alométrico e de Fulton, índice de alteração histopatológica, assim como dos parâmetros de qualidade de água, foram submetidos às análises estatísticas ANOVA (Analysis of Variance) ou Kruskal-Wallis, e aos testes de Tukey ou Dunn, considerando uma significância mínima de 5,0\% $(\mathrm{p}=0,05)$. Para isso, utilizou-se o programa BioEstat 5.0. Foram feitos os testes de Lilliefors e Shapiro-Wilk para a verificação da normalidade entre os dados. Também, foi cumprida a homocedasticidade, condição exigida para tais análises. 


\section{RESULTADOS E DISCUSSÃO}

Na Tabela 3, apresentam-se os resultados médios e respectivos desvios padrão e níveis de significância dos parâmetros de qualidade das águas utilizadas nos três tratamentos.

A faixa de $\mathrm{pH}$ considerada ótima para o cultivo de organismos aquáticos, inclusive em sistemas de reúso, varia de 6 a 9 (Colt, 2006). Observa-se que os valores de $\mathrm{pH}$ nos dois tratamentos com o uso de esgoto tratado (para ET, $\mathrm{pH}=8,25$; para $\mathrm{ED}, \mathrm{pH}=8,38$ ) ficaram dentro da faixa considerada ótima. Já no tratamento que foi abastecido com água bruta de um poço freático, o valor médio de $\mathrm{pH}(9,57)$ ficou acima da faixa ótima, provavelmente devido à característica do subsolo da região.

Tabela 3. Resultados (média \pm desvio padrão), nível de significância e número de amostras dos parâmetros físico-químicos de qualidade das águas de cultivo

\begin{tabular}{|c|c|c|c|c|c|}
\hline \multirow[t]{2}{*}{ Parâmetros } & \multicolumn{3}{|c|}{ Tratamentos experimentais } & \multirow[t]{2}{*}{$\mathrm{P}$} & \multirow[t]{2}{*}{$\mathrm{n}$} \\
\hline & ET & $\mathrm{ED}$ & $\mathrm{AB}$ & & \\
\hline Temperatura $\left({ }^{\circ} \mathrm{C}\right)$ & $27,0 \pm 1,0 b$ & $27,3 \pm 1,0 a b$ & $28,6 \pm 1,2^{a}$ & 0,0336 & 07 \\
\hline $\mathrm{OD}\left(\mathrm{mg} \mathrm{O}_{2} \mathrm{~L}^{-1}\right)$ & $1,29 \pm 1,10 b$ & $2,06 \pm 1,61 b$ & $8,96 \pm 1,92^{a}$ & 0,0001 & 10 \\
\hline $\mathrm{CE}\left(\mu \mathrm{S} \mathrm{cm}{ }^{-1}\right)$ & $927 \pm 149 a$ & $611 \pm 102 b$ & $383 \pm 56 c$ & 0,0001 & 05 \\
\hline Cloreto (mg L $\left.{ }^{-1}\right)$ & $212,7 \pm 11,1 a$ & $127,0 \pm 3,2 b$ & $63,7 \pm 3,2 c$ & 0,0001 & 05 \\
\hline $\mathrm{AT}\left(\mathrm{mg} \mathrm{CaCO} \mathrm{L}^{-1}\right)$ & $206,4 \pm 20,4 a$ & $147,6 \pm 23,5 b$ & $117,1 \pm 30,8 b$ & 0,0006 & 05 \\
\hline $\mathrm{DT}\left(\mathrm{mg} \mathrm{CaCO} \mathrm{L}^{-1}\right)$ & $24,1 \pm 2,0 a$ & $17,4 \pm 1,9 b$ & $8,0 \pm 1,4 c$ & 0,0001 & 05 \\
\hline $\mathrm{pH}$ & $8,61 \pm 0,61 a b$ & $8,53 \pm 0,42 b$ & $9,68 \pm 0,18 a$ & 0,0027 & 07 \\
\hline $\mathrm{NAT}\left(\mathrm{mg} \mathrm{L}^{-1}\right)$ & $2,65 \pm 0,42 a$ & $0,87 \pm 0,38 b$ & $0,41 \pm 0,16 b$ & 0,0001 & 06 \\
\hline $\mathrm{N}-\mathrm{NH}_{3}\left(\mathrm{mg} \mathrm{L}^{-1}\right)$ & $0,62 \pm 0,53 a$ & $0,22 \pm 0,20 a$ & $0,32 \pm 0,13 a$ & 0,1034 & 06 \\
\hline Nitrito $\left(\mathrm{mg} \mathrm{L}^{-1}\right)$ & $1,04 \pm 0,72 a$ & $0,64 \pm 0,49 a b$ & $0,04 \pm 0,05 b$ & 0,0133 & 07 \\
\hline Nitrato $\left(\mathrm{mg} \mathrm{L}^{-1}\right)$ & $0,20 \pm 0,17 a$ & $0,13 \pm 0,11 a$ & $0,11 \pm 0,09^{\mathrm{a}}$ & 0,6886 & 05 \\
\hline $\mathrm{DQO}\left(\mathrm{mg} \mathrm{O}_{2} \mathrm{~L}^{-1}\right)$ & $175,77 \pm 42,10 a$ & $115,67 \pm 37,35 a b$ & $96,36 \pm 43,77 b$ & 0,0116 & 06 \\
\hline
\end{tabular}

OD: oxigênio dissolvido; CE: condutividade elétrica; AT: alcalinidade total; DT: dureza total; NAT: nitrogênio amoniacal total; N-NH3: amônia não ionizada; DQO: demanda química de oxigênio; ET: esgoto tratado; ED: egoto diluído; $\mathrm{AB}$ : água bruta; p: nível de significância; n: número de amostras. Letras diferentes relativas a um mesmo parâmetro expressam diferença estatisticamente significativa.

Boyd e Tucker (1998) indicam a faixa ótima de temperatura para o cultivo de peixes tropicais entre 20 e $30^{\circ} \mathrm{C}$; já Küçük (2010), cultivando Poecilia velifera, obteve melhores resultados em temperatura de $25^{\circ} \mathrm{C}$, em comparação a $30^{\circ} \mathrm{C}$. Nesta pesquisa, a temperatura média dos tratamentos ficou dentro da faixa considerada ideal.

Os valores de condutividade elétrica para aquicultura, segundo Boyd e Tucker (1998), devem ser menores que $1.000 \mu \mathrm{S} \mathrm{m}^{-1}$. Assim, é possível observar que apenas no final do período de cultivo esse valor foi ultrapassado somente no tratamento ET.

Segundo Colt (2006), a toxicidade da amônia é geralmente associada à concentração da amônia não ionizada $\left(\mathrm{NH}_{3}\right)$, devido à sua capacidade de se mover através das membranas celulares. As concentrações de amônia não ionizada nos três tratamentos ficaram acima de $0,1 \mathrm{mg} / \mathrm{L}$, valor proposto por Boyd e Tucker (1998) para exposições prolongadas.

Colt (2006) recomenda concentrações de oxigênio dissolvido (OD), em sistemas de reúso, acima de 3,5mg/L para espécies rústicas. Apenas o tratamento ET apresentou média de OD $(3,09 \mathrm{mg} / \mathrm{L})$ abaixo do valor de referência. O valor médio de DQO no tratamento ET foi de $168,53 \mathrm{mg} / \mathrm{L}$, o que justifica a baixa concentração de OD.

Na Tabela 4 podem-se observar os resultados médios e respectivos desvios padrão dos parâmetros zootécnicos obtidos na avaliação de rendimento dos cultivos. Para todos os parâmetros de rendimento zootécnico avaliados, o tratamento abastecido com esgoto tratado diluído foi o que apresentou os melhores resultados, indicando que o esgoto doméstico tratado tem capacidade de sustentar o desenvolvimento da espécie testada nas condições de cultivo. 
Tabela 4. Duração do cultivo (dias), resultados (média \pm desvio padrão) e nível de significância (p) dos parâmetros zootécnicos obtidos no final do cultivo

\begin{tabular}{|c|c|c|c|c|}
\hline \multirow[t]{2}{*}{ Parâmetros Zootécnicos } & \multicolumn{3}{|c|}{ Tratamentos Experimentais } & \multirow[t]{2}{*}{$\mathrm{p}$} \\
\hline & ET & ED & $\mathrm{AB}$ & \\
\hline Dias de cultivo & 56 & 56 & 56 & - \\
\hline Comprimento final (mm) & $27,9 \pm 4,11 c$ & $35,2 \pm 5,57 a$ & $30,5 \pm 5,57 b$ & 0,0001 \\
\hline Crescimento (mm) & $15,3 \pm 4,11 c$ & $22,7 \pm 5,57 a$ & $18,5 \pm 5,57 b$ & 0,0001 \\
\hline $\mathrm{CD}\left(\mathrm{mm} \mathrm{dia}{ }^{-1}\right)$ & $0,273 \pm 0,073 b$ & $0,397 \pm 0,115 a$ & $0,323 \pm 0,110 b$ & 0,0001 \\
\hline Peso final (g) & $0,439 \pm 0,091 b$ & $0,592 \pm 0,043 a$ & $0,269 \pm 0,048 b$ & 0,0001 \\
\hline Ganho de Peso (g) & $0,407 \pm 0,091 b$ & $0,559 \pm 0,043^{\mathrm{a}}$ & $0,237 \pm 0,048 b$ & 0,0001 \\
\hline GPD $\left(\mathrm{g} \mathrm{dia}^{-1}\right)$ & $0,007 \pm 0,002 b$ & $0,010 \pm 0,001 a$ & $0,004 \pm 0,001 c$ & 0,0001 \\
\hline TCE (\% do peso dia $\left.^{-1}\right)$ & $4,63 \pm 0,44 b$ & $5,15 \pm 0,13 a$ & $3,78 \pm 0,33 b$ & 0,0001 \\
\hline Taxa de sobrevivência (\%) & $22,0 \pm 0,31 c$ & $95,9 \pm 14,79 a$ & $62,5 \pm 9,06 b$ & - \\
\hline
\end{tabular}

São poucos, ou mesmo inexistentes, os trabalhos sobre cultivo de molinésias em esgoto tratado, para fins de comparação com a presente pesquisa. Küçük (2010), cultivando Poecilia velifera, obteve ganho de peso diário variando de $0,0062 \mathrm{~g} /$ dia a 0,0107g/dia, para águas com diferentes características - temperatura, dureza, alcalinidade, cálcio, magnésio, bicabornato, amônia, nitrito, nitrato, condutividade elétrica. Pode-se observar que os valores citados foram semelhantes aos alcançados neste experimento.

Observa-se que o tratamento ED foi o que apresentou a maior taxa de sobrevivência, com média de $95,9 \pm 12,07 \%$, seguido pelo tratamento $\mathrm{AB}$, com $62,5 \pm 7,40 \%$, e pelo tratamento $\mathrm{ET}$, com 22,0 $\pm 0,25 \%$. Apenas os valores obtidos no tratamento ET ficaram abaixo dos alcançados por
Küçük (2010), que obteve taxas de sobrevivência variando de $33,3 \%$ a $55,6 \%$. Alguns fatores podem ter causado a mortalidade dos peixes, dentre os quais é válido salientar o desequilíbrio na relação alcalinidade total:dureza total, que ocasionou a redução do poder tampão, com consequente elevação do $\mathrm{pH}$, que, por sua vez, contribuiu para aumentar a concentração e toxicidade da amônia não ionizada para níveis subletais. A baixa concentração de oxigênio dissolvido também pode ter contribuído para a mortalidade.

Quanto à avaliação do bem-estar animal, na Tabela 5 constam os parâmetros da relação peso:comprimento, as equações geradas, os tipos de crescimento e os resultados médios para os fatores de condição alométrico e de Fulton.

Tabela 5. Tamanho das amostras utilizadas, parâmetros da relação peso:comprimento, equações geradas, tipos de crescimento e resultados (média desvio padrão das amostras de peixes) dos fatores de condição (K) avaliados na pesquisa

\begin{tabular}{lccc}
\hline \multicolumn{1}{c}{ Parâmetros } & ET & ED & AB \\
\hline $\mathrm{N}$ & 33 & 37 & 33 \\
$\mathrm{~A}$ & 0,0225 & 0,0225 & 0,0296 \\
$\Theta$ & 2,8720 & 2,4947 & 2,1654 \\
Equação (P:C) & $\mathrm{P}=0,0225 \times \mathrm{C}^{2,8720}$ & $\mathrm{P}=0,0270 \times \mathrm{C}^{2,4947}$ & $\mathrm{P}=0,0296 \times \mathrm{C}^{2,1654}$ \\
Crescimento & Alométrico negativo & Alométrico negativo & Alométrico negativo \\
K-Fulton & $2,19 \pm 0,90^{a}$ & $1,76 \pm 0,71 a$ & $1,83 \pm 0,65 a$ \\
K-alométrico & $2,39 \pm 0,95 b$ & $2,86 \pm 1,01 a b$ & $3,10 \pm 0,92 a$ \\
\hline n = tamanho da amostra; A = intercepto; $\theta:$ coeficiente de Alometria; K = fator de condição; $\mathrm{P}=$ peso $(\mathrm{g}) ; \mathrm{C}=$ \\
comprimento (cm); ET: esgoto tratado; ED: esgoto tratado diluído; AB: água bruta. Letras diferentes relativas a um \\
mesmo parâmetro expressam diferença estatisticamente significativa.
\end{tabular}

$\mathrm{O}$ coeficiente de alometria - b $(\theta)$ apresentou valores de 2,872 no tratamento ET, 2,4947 no tratamento ED e 2,1654 no tratamento AB. Com exceção deste último, os valores obtidos ficaram dentro dos recomendados pela literatura. Segundo Oscoz et al. (2005), todas as estimativas dos valores de " $\theta$ " devem estar na faixa de 2,5 a 3,5 . 
Para valores de " $\theta$ " menores que 3,0 , o crescimento é considerado alométrico negativo, isto é, o peixe cresce mais que ganha peso. Esse comportamento foi observado, especialmente, para o tratamento $\mathrm{AB}$.

O fator de condição (K) é um índice muito utilizado em estudos de biologia pesqueira, pois indica o grau de bem-estar do peixe frente ao meio em que vive, devendo permanecer constante, independentemente do tamanho que o peixe possa vir a ter em um determinado momento (Gomiero e Braga, 2003).

Para o fator de condição de Fulton $\left(\mathrm{K}_{\text {Fulton }}\right)$, os tratamentos experimentais não apresentaram diferença estatisticamente significativa; no entanto, numericamente, o tratamento ET foi o de maior valor $(2,19 \pm 0,90)$, seguido pelo tratamento $\mathrm{AB}(1,83 \pm 0,65)$ e pelo tratamento ED (1,76 \pm 0,71). Kuçuk (2010), cultivando Poecilia velifera em diferentes padrões de qualidade de água, obteve valores do fator de condição de Fulton $\left(\mathrm{K}_{\text {Fulton }}\right)$ de 1,$45 ; 1,47 ; 1,54 \mathrm{e}$ 1,61 , em quatro tratamentos experimentais.

O coeficiente de alometria $(\theta)$ é aplicado na equação do fator de condição alométrico $\left(\mathrm{K}_{\mathrm{alom}}\right)$ para adaptar o fator ao crescimento da espécie em estudo, conforme as condições experimentais. Com a aplicação desse coeficiente, deixa-se de usar a constante de Fulton (3,0 - admitindo crescimento isométrico), que considera o crescimento isométrico para peixes em geral.

Quanto ao $\mathrm{K}_{\text {alom }}$ o tratamento $\mathrm{AB}$ apresentou o maior resultado entre os tratamentos experimentais $(3,10 \pm 0,92)$, seguido pelo tratamento ED $(2,86 \pm 1,01)$ e pelo tratamento ET $(2,39 \pm 0,95)$. É valido salientar que o tratamento AB apresentou diferença estatisticamente significativa apenas quando comparado ao ET.
Le Cren (1951) explica que os resultados do fator de condição são de difícil interpretação quando ocorre diferença entre as variáveis peso e comprimento das amostras avaliadas. Quando se tem um mesmo comprimento e variação apenas no peso, a comparação entre os resultados é fácil.

No presente estudo, os resultados de peso e comprimento apresentaram-se diferentes entre si, inclusive sendo as diferenças estatisticamente significativas. Dessa forma, a comparação direta dos resultados do fator de condição tornou-se difícil.

Ao se tratar de fator de condição, a principal referência científica é o trabalho realizado por Le Cren (1951). Esse autor cita que a "condição" de um peixe reflete as recentes características físicas e biológicas do ambiente, que influenciam, entre outros fatores, as condições alimentares. Dessa forma, o fator de condição pode ser usado como indicador da condição ambiental, podendo ser avaliado conjuntamente com os indicadores físico-químicos de qualidade de água. É possível apontar que o conjunto dos fatores avaliados indica $\mathrm{o}$ tratamento ED como o que proporcionou as melhores condições ambientais de cultivo, fato que resultou nos melhores resultados zootécnicos observados.

Não foram encontradas referências sobre fator de condição alométrico $\left(\mathrm{K}_{\text {alom }}\right)$ para a espécie em estudo, não sendo possível a realização das comparações e avaliações pertinentes.

Excetuando-se as brânquias e rins, não foram observadas alterações significativas em nenhum outro órgão dos peixes cultivados nos três tratamentos experimentais. $\mathrm{Na}$ Tabela 6 podem ser observados os valores obtidos por meio do índice de alteração dos tecidos para as brânquias e rins dos três tratamentos experimentais.

Tabela 6. Resultados (média \pm desvio padrão) dos índices de alteração, segundo Bernet et al. (1999), das brânquias e rins atribuídos por meio da avaliação histopatológica dos peixes dos três tratamentos experimentais

\begin{tabular}{lcccc} 
& \multicolumn{3}{c}{ Tratamentos Experimentais } & $\mathrm{p}$ \\
\cline { 2 - 4 } \multicolumn{1}{c}{ Órgãos } & $\mathrm{ET}$ & $\mathrm{ED}$ & $\mathrm{AB}$ & 0,0081 \\
Brânquias & $10,6 \pm 5,0 b(\mathrm{n}=9)$ & $10,4 \pm 4,1 b(\mathrm{n}=7)$ & $16,0 \pm 4,3(\mathrm{n}=7)$ & 0,0173 \\
Rins & $21,7 \pm 7,0 a(\mathrm{n}=10)$ & $17,0 \pm 7,7 a b(\mathrm{n}=8)$ & $12,7 \pm 3,6 b(\mathrm{n}=9)$ & 0,0173 \\
\hline
\end{tabular}

ET: esgoto tratado; ED: esgoto diluído $\mathrm{AB}$ : água bruta. n: número de amostras. Letras diferentes relativas a um mesmo parâmetro expressam diferença estatisticamente significativa. 
Observa-se que, nas brânquias, o valor do índice de alteração do tecido foi maior no tratamento $\mathrm{AB}$ que nos demais, inclusive apresentando diferença estatisticamente significativa para $\mathrm{p}<$ 0,05 .

Já na avaliação histopatológica dos rins, o índice de alteração apresentou maior resultado nos peixes do tratamento ET, sendo a diferença estatisticamente significativa apenas em relação ao tratamento $\mathrm{AB}$. O segundo maior resultado foi obtido nos peixes do tratamento ED e o menor, no tratamento $\mathrm{AB}$, não havendo diferença estatisticamente significativa entre estes dois últimos.

A Figura 2 permite a visualização de algumas das alterações estruturais encontradas no presente estudo. Na Figura 2A é possível visualizar um filamento branquial primário no tratamento $\mathrm{AB}$, com levantamento do epitélio, hiperplasia dos ionócitos e fusão das lamelas secundárias. Na Figura 2B visualiza-se uma fotomicrografia da estrutura renal de um peixe do tratamento ET, onde são destacados tubos renais com oclusão, hipertrofia e vacuolização do epitélio desses tubos e hipertrofia do glomérulo com correspondente diminuição do espaço de Bowman.

As alterações histopatológicas podem ser usadas como indicadores de poluentes antropogênicos sobre os organismos, e são um reflexo da saúde da população presente em um determinado ecossistema. Estudos anteriores relataram que a exposição de peixes aos poluentes (agrícola, industrial e esgoto) resulta em várias alterações patológicas em diferentes tecidos de peixes (Mohamed, 2009).
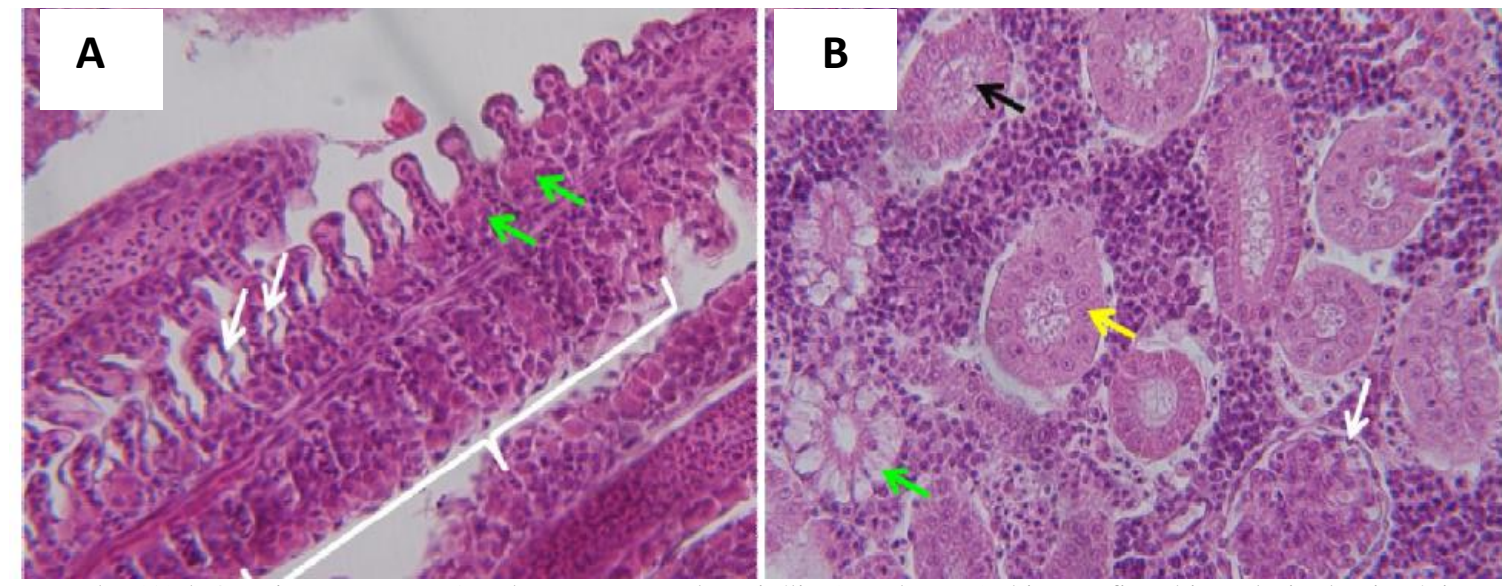

Legenda: (A) brânquia tratamento $\mathrm{AB}$; levantamento do epitélio (seta branca); hipertrofia e hiperplasia dos ionócitos (seta verde); fusão lamelar (chave branca). (B) rim tratamento ET; hipertrofia glomerular e diminuição do espaço de Bowman (seta branca); túbulo com núcleo celular hipertrofiado (seta amarela); túbulo com vacuolização das células epiteliais (seta verde); oclusão do túbulo renal e degeneração do epitélio (seta preta).

Figura 2. Amostras de alterações nos tecidos utilizados na avaliação hitopatológica dos peixes cultivados: (A) tecido branquial de peixe do tratamento $\mathrm{AB}$; (B) tecido renal de peixe do tratamento $\mathrm{ET}$.

Alterações como levantamento epitelial, hiperplasia e hipertrofia das células do epitélio, além de fusão lamelar, são exemplos de mecanismo de defesa, por meio do aumento da distância entre o ambiente externo e o sangue, impedindo a entrada de contaminantes (Camargo e Martinez, 2007).

Levantamento do epitélio lamelar é uma das primeiras alterações a ocorrerem nas brânquias dos peixes mediante ação aguda de substâncias tóxicas como óleos, detergentes, metais pesados e amônia (Nascimento et al., 2012). Óleos e detergentes são componentes comuns dos esgotos domésticos. Os resultados de amônia, neste experimento, foram elevados, especialmente no tratamento ET (Nitrogênio Amoniacal Total $=2,65 \mathrm{mg} \mathrm{L}^{-1} ; \mathrm{NH}_{3}=0,62 \mathrm{mg} \mathrm{L}$ $\left.{ }^{1}\right)$.

Os rins constituem os primeiros órgãos dos peixes a serem afetados por contaminantes 
presentes na água (Thophon et al., 2003). Apesar das alterações histopatológicas nos rins de peixes não serem associadas a um agente estressor específico, Veiga et al. (2002) relatam que peixes expostos a contaminantes orgânicos apresentam degeneração tubular, com inchaço, embaçamento e vazamento do hialino; redução do espaço de Bowman e dilatação dos capilares dos glomérulos. As alterações histopatológicas observadas na presente pesquisa afetaram o rendimento zootécnico dos peixes cultivados, no entanto não inviabilizaram o cultivo da espécie em estudo, pois não podem ser diretamente relacionadas com a mortalidade observada. Um resultado de relevante importância observado na presente pesquisa foi $\mathrm{o}$ fato de não ter sido constatada ocorrência de vermes ou de outros parasitas nos músculos dos peixes analisados. Esse resultado complementa os obtidos por outras pesquisas realizadas com tilápias do Nilo, Oreochromis niloticus (Santos et al., 2009; Monteiro et al., 2011).

Não foram notadas ulcerações na pele, descamação, despigmentação, ou qualquer tipo de necrose significativa no corpo ou nadadeiras dos peixes nos três tratamentos testados.

\section{CONCLUSÕES}

Observou-se que o esgoto doméstico tratado em lagoas de estabilização em série, para as condições da pesquisa, é adequado para o cultivo de Poecilia sp., quando diluído em água bruta, em partes iguais. $O$ tratamento que utilizou esgoto doméstico tratado diluído em água (50\% de cada parte) foi o que promoveu o melhor desempenho zootécnico dos peixes ornamentais da espécie Poecilia sp.. Dentre os fatores utilizados como indicadores de bem-estar animal, apenas a avaliação histopatológica foi capaz de proporcionar resultado conclusivo, apontando o tratamento abastecido com esgoto tratado diluído como o que proporcionou o ambiente mais confortável para o desenvolvimento dos peixes cultivados. As características ornamentais dos peixes cultivados não sofreram influência do uso do esgoto doméstico tratado no abastecimento dos tanques experimentais, pois não foi observada qualquer alteração nos padrões de coloração e formato de nadadeiras, nem sinais de ulceração, descoloração, descamação ou máformação nos peixes.

\section{REFERÊNCIAS}

ALBERT, A. Selective Toxicity. London: Chapman and Hall, 1973, 597 p.

ALMEIDA, S.C.A.; NUÑER, A.P.O. Crescimento de Pimelodus maculatus (Actinopterygii, Pimelodidae) estocados em diferentes densidades em tanques-rede. Biotemas. v.22, p.113-119, 2009.

APHA - American Public Health Association. Standard Methods for the examination of water and wastewater. 19.ed. Washington, DC: APHA/AWWA - WPCF, 2005.

BERNET, D.; SCHMIDT, H.; MEIER, W. et al. Histopathology in fish: proposal for a protocol to assess aquatic pollution. J. Fish Dis., v.22, p.2534, 1999.

BOYD, C.E.; TUCKER, C.S. Pond aquaculture water quality management. Norwell, MA, USA: Kluwer Academic Publishers, 1998, 631p

BUNTING, S.W. Confronting the realities of wastewater aquaculture in peri-urban Kolkata with bioeconomic modeling. Water Res., v.41, p.499-505, 2007.

CAMARGO. M.M.P.; MARTINEZ, C.B.R. Histopathology of gills, kidney and liver of a Neotropical fish caged in an urban stream. Neotrop. Ichthyol., v.5, p.327-336, 2007

COLT, J. Water quality requirements for reuse systems. Aquac. Eng., v.34, p.143-156, 2006.

EMERSON, K.R.; RUSSO, R.C.; LUND, R.E. et al. Aqueous ammonia equilibrium calculations: effect of $\mathrm{pH}$ and temperature. J. Fish. Res. Board Can., v.32, p.2377- 2383, 1975.

GOMIERO, L.M.; BRAGA, F.M.S. Relação peso:comprimento e fator de condição para Cichla cf. ocellaris e Cichla monoculus (Perciformes, Cichlidae) no reservatório de Volta Grande, rio Grande - MG/SP. Acta Sci., Biol. Sci., v.25, p.79-86, 2003.

KÜÇÜK, S. The effects of water type on growth, survival and condition of Poecilia velifera. Afr. J. Biotechnol., v.9, p.760-763, 2010.

LE CREN, E.D. The length-weight relationship and seasonal cycle in gonadal weight condition in the perch (Perca fluvialitis). J. Anim. Ecol., v.20, p.201-219, 1951 . 
LEMOS, J.R.G.; TAVARES-DIAS, M.; MARCON, J.L. et al. Relação peso:comprimento e fator de condição em espécies de peixes ornamentais do Rio Negro, Estado do Amazonas (Brasil). In: CONGRESO IBEROAMERICANO VIRTUAL DE ACUICULTURA, 4., 2006. Anais... Disponível em: http://www.civa2006.org, p.721-725. Acessado em:

MOHAMED, F.A.S. Histopathological Studies on Tilapia zillii and Solea vulgaris from Lake Qarun, Egypt. World J. Fish \& Marine Sci., v.1, p.29-39, 2009.

MONTEIRO, C.A.B.; SANTOS, E.S.; MOTA, S. et al. Efeito da aeração por air-lift na alevinagem de tilápia do Nilo em esgoto doméstico tratado. Revista DAE, v.186, p.16-22, 2011.

NASCIMENTO, A.A.; ARAÚJO, F.G.; GOMES, I.D. et al. Fish Gills Alterations as Potential Biomarkers of Environmental Quality in a Eutrophized Tropical River in South-Eastern Brazil. J. Vet. Med., v.1, p.1-8, 2012.

OSCOZ, J.; CAMPOS, F.; ESCALA, M.C. Weight-length relationships of some fish species of the Iberian Peninsula. J. appl. ichthyol., v.21, p.73-74, 2005.

PIEDRAS, S.R.N.; MORAES, P.R.R.; POUEY, J.L.O.F. Desempenho de juvenis de catfish (Ictalurus punctatus) em diferentes temperaturas. R. Bras. Agrociência, v.12, p.367-370, 2006.
SANTOS, A.F.G.N.; SANTOS, L.N.; ARAÚJO, F.G. et al. Relação peso:comprimento e fator de condição do acará, Geophagus brasiliensis, no reservatório de Lajes, RJ. Rev. Univ. Fed. Rural, Série Ciências da Vida, v.22, p.115-121, 2002.

SANTOS, E.S.; OLIVEIRA, M.A.; MOTA. S. et al. Crescimento e qualidade dos alevinos de tilápia do Nilo produzidos em esgoto doméstico tratado. Rev. Cienc. Agron., v.40, p.232-239, 2009.

SAXBY, A.; ADAMSA, L.; SNELLGROVEB, D. et al. The effect of group size on the behaviour and welfare of four fish species commonly kept in home aquaria. Appl. anim. behav. sci., v.125, p.195-205, 2010.

SUDHA, C. Study on induced breeding in ornamental fish, poecilia sphenops. Eur. J. Exp. Biol., v.2, p.1250-1255, 2012.

THOPHON, S.; KRUATRACHUE, V.; UPATHAN, E.S. et al. Histopathological alterations of white seabass, Lates calcarifer in acute and subchronic cadmium exposure. Environ. Pollut., v.121, p.307-320, 2003.

VEIGA, M.L.; RODRIGUES, E.L.; PACHECO, F.J. et al. Histopathologic changes in the kidney tissue of Prochilodus lineatus, 1836 (Characiformes, Prochilodontidae) induced by sublethal concentration of Trichlorfon exposure. Braz. Arch. Biol. Technol., v.45, p.171-175, 2002. 